\title{
BIO's plan to promote opportunity and access for the underserved
}

\begin{abstract}
To the Editor — On Memorial Day this year, the senseless murder of another unarmed black man in Minneapolis, Minnesota, touched off days of protest against police brutality and racial inequality. In the ensuing months, disturbing videos of George Floyd and other minorities killed and mistreated by police have filled our television screens and stirred the American conscience. Meanwhile, the worst pandemic in a century rages unchecked across the globe, hitting communities of color the hardest because COVID-19 preys on the immunocompromised in a time when too many African Americans struggle to access quality healthcare.
\end{abstract}

The world is waking up to the reality that this new, cutting-edge industry called biotechnology is humanity's best and only hope to eradicate the COVID-19 threat. Biotechnology is also the most powerful tool we have to broaden access to clean air and water, nutritious foods, breakthrough medicines and sustainably made consumer products.

At this galvanizing moment of national awakening, the Biotechnology Innovation Organization (BIO) is challenging our industry and its members to walk the walk of racial equity in hiring and promotions, clinical trial enrollment and the bioeconomy.

We believe that the biotech industry can not only save lives, but also advance equal opportunity and social justice by creating more entry points for minority scientists and more advancement into leadership positions. We also have an obligation to make sure our transformative scientific breakthroughs are accessible and affordable to under-represented communities.

BIO recognizes that these are not overnight fixes and that to be successful we need an actionable plan. So last month, as the world's largest biotechnology advocacy association, we rolled out our BIOEquality Agenda. We are leading a national effort in collaboration with our partners and member organizations in the biotechnology sector to support stronger and healthier communities. The effort will be organized around three pillars (Box 1): health equity, investment in current and future researchers, and expanded opportunities for women and other under-represented populations.

\section{Box 1 | The BIOEquality Agenda}

BIO's efforts to advance equal opportunity, social justice, and product accessibility and affordability center around three pillars:

\section{Promote health equity}

- Enhance clinical trial diversity by partnering with contract research organizations and minority-serving institutions

- Promote access to vaccines and therapeutics for uninsured and underserved populations, especially related to COVID-19

- Foster enhanced nutritional, environmental and mental wellness opportunities in economically disadvantaged communities

Invest in the current and next generation of scientists

- Inventory and promote visibility of industry-sponsored minority training programs

I took over as BIO's president and CEO on 1 June of this year. A decade earlier, I was a newly appointed associate science director at the US Food and Drug Administration (FDA) when the Office of Minority Health was launched. A white paper at that time noted that African Americans represented $12 \%$ of the US population, but only $5 \%$ of clinical trial participants. Hispanics were $16 \%$ of the population, but only $1 \%$ of trial participants.

Ten years later, we continue to see substantial under-representation in phase 3 trials, where thousands must enroll before a medicine is approved. According to a disheartening report press-released by Tufts University, although the level of data available on participant representation by sex and age showed notable improvement from 2007 to 2017 , participant representation by race and ethnicity did not. Black participants were under-represented by more than $20 \%$ relative to proportionate levels in clinical trials for $80 \%$ of all drugs and biologics approved by the FDA during 2007-2017.
- Establish STEM-training best practices for under-represented populations

- Create a network of minority scientists and engineers

- Facilitate a partnering network, including hiring of industry-trained minority scientists and engineers

Expand opportunity for women and other under-represented populations

- Enhance use of minority- and women-owned small businesses in biotech supply chains to promote economic development of marginalized communities, especially those hardest hit by COVID-19

- Promote diversity within the US National Institutes of Health's Small Business Innovation Research grants to increase opportunity for minority entrepreneurship in the life sciences

Hispanic or Latinx were under-represented by $62 \%$.

BIO has already begun working with organizations outside our industry who really understand these under-represented communities, including the National Association of Black Churches and the National Urban League. Together, we will develop and implement strategies that seek to better educate those who enroll participants and build bridges in the African American community.

There is an understandable legacy of mistrust when it comes to medical testing and trials. The Tuskegee study on syphilis in black men took place without participants' informed consent for more than 40 years while many went untreated. Even once it became clear that penicillin was effective, many were not given adequate treatment for their disease.

To make sure the treatments for COVID19 and other illnesses are safe and effective on members of minority and vulnerable populations, it is important to have their participation in clinical trials. So we need to 
build confidence, have an open and honest dialogue about the past, and work with local community leaders who can be that trusted voice.

While the BIOEquality Agenda will address inequities in healthcare delivery, STEM (science, technology, engineering and math) programs and minority entrepreneurship, BIOEquality complements the Right Mix Matters initiative we launched last year to accelerate gender, racial, ethnic and LGBTQ (lesbian, gay, bisexual, transgender and queer or questioning) representation on biotech company boards, in C-suites and in functional leadership positions.

$\mathrm{BIO}$ is deeply committed to both BIOEquality and the Right Mix Matters campaign. In these difficult and uncertain times, we will continue to build support for a more equitable future for the biotech sector and the millions of patients across the world who depend on our success.
Michelle McMurry-Heath $\bowtie$

Biotechnology Innovation Organization,

Washington, DC, USA.

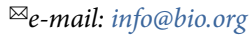

Published online: 11 September 2020

https://doi.org/10.1038/s41587-020-0688-8

Competing interests

M.M.-H. works for industry trade organization the Biotechnology Innovation Organization (BIO). She was employed by Johnson \& Johnson and holds stock in Johnson \& Johnson.

\section{Leveling up citizen science}

To the Editor - Over the past decade, online science discovery games (SDGs) have become an increasingly popular practice for engaging the public in research activities. This methodology has had the most impact in molecular and cell biology, where participants are recruited for not only data collection and classification tasks, but also solving complex computational problems that require human supervision or insight. As the life sciences move toward increasingly large and ambitious projects, however, there is a need to complement existing models that try to attract users to yet another third-party website. Here we describe our experience with Borderlands, a massively multiplayer online game that embeds citizen-science problems into its virtual universe and engages a new user base, with implications for future citizen science initiatives seeking to meet the growing demands of biology.

\section{Citizen science in biology}

Since 2008, the Foldit team has recruited online participants to predict protein $3 \mathrm{D}$ structure via a $3 \mathrm{D}$ puzzle game distributed as a standalone program for personal computers $^{1}$. This project helped refine a retroviral protease structure ${ }^{2}$ and later discovered novel protein folds ${ }^{3}$. Two years later, $\mathrm{Phylo}^{4}$ pushed the gamification concept to reach a broader public by crowdsourcing comparative genomics tasks via tile-matching puzzle games. Other successful projects include web-based games for RNA structural modeling (for example, EteRNA $^{5}$ ) and neuron segmentation (for example, EyeWire ${ }^{6}$.

The efficiency and effectiveness of SDGs is necessarily determined by the number of contributors and volume of data collected, regardless of citizen-science strategy. Games

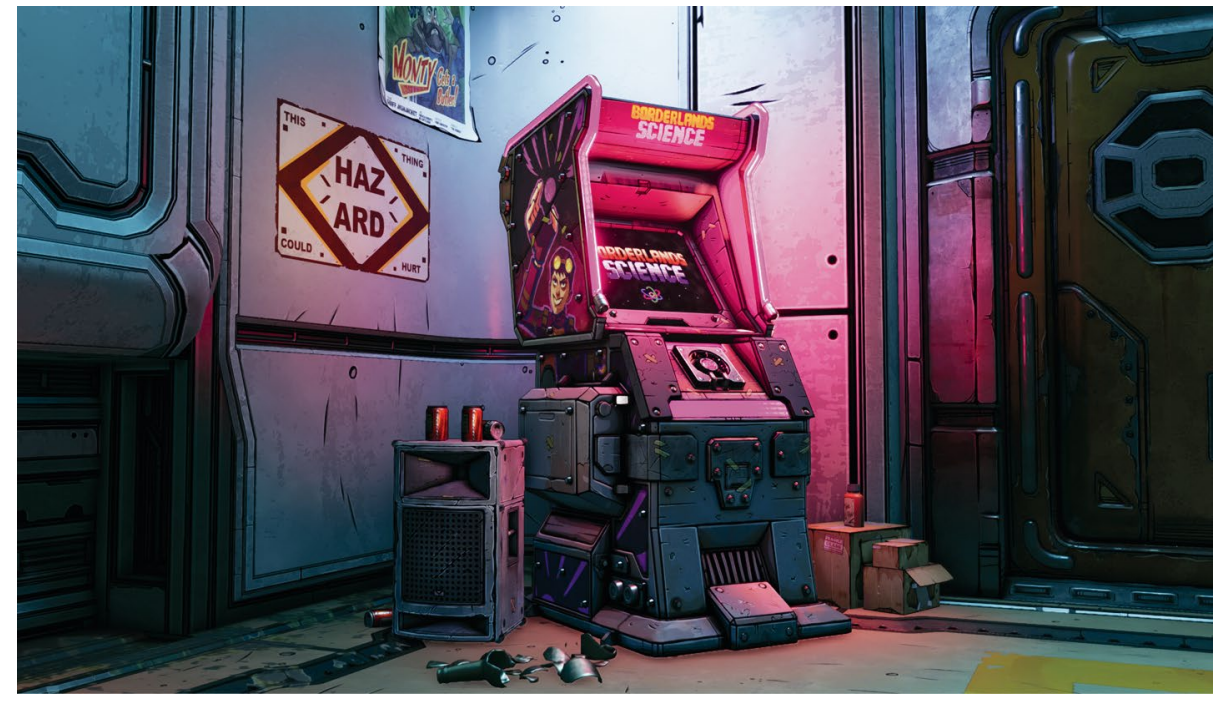

Fig. 1 | Arcade booth allowing the gamers to access Borderlands Science in Borderlands 3.

like Foldit take advantage of large groups of participants for identifying skilled users and promising solutions to a hard problem. In contrast, Phylo and related SDGs aim to harness the wisdom of the crowd by detecting collective behaviors of the gamers while solving small instances of the problem, and using this knowledge to build a global solution.

Having sustainable and large crowds of participants is necessary for opening this technology as a service to the whole scientific community $^{7}$. Popular SDGs represent the best option for solving on-demand problems for many groups of independent scientists. Although few such platforms have yet to be deployed, this approach constitutes a natural and promising evolution of SDGs that is exemplified by recent initiatives like
OpenVaccine or OpenCRISPR from the EteRNA project.

Recruiting large groups of volunteers should also serve long-term objectives and not be confined to solving a specific problem without a larger context. Projects like ENCODE $^{8}$ or the Earth Microbiome Project ${ }^{9}$ involve hundreds of scientists worldwide and require assembly and coordination of many diverse areas of expertise. At all stages of the research pipeline and at various degrees of involvement, collection, curation and analysis of the data need the supervision of human experts. But as the size and complexity of the project teams expand, so too does the cost of human intervention and logistics, which may not be sustainable. To expand the boundaries of the system, we need to refactor the organization of large scientific projects. 